\title{
Patient Satisfaction in a Culturally Diverse Environment: A Case Study of Dubai
}

\author{
Asima Shirazi* and Dr. Amanat Ali Jalbani \\ SZABIST \\ Karachi, Pakistan
}

\begin{abstract}
The purpose of undertaking this study is to identify the factors that are taken into account when patients select doctors and to determine the variables that increase or decrease the possibility of a person being satisfied with his or her doctor. The analysis was based on a survey of residents in Dubai.
\end{abstract}

\section{INTRODUCTION}

Patient satisfaction is an important element in assessing the effectiveness of a healthcare system. In a culturally diverse society, such as Dubai, both the health care providers and users come from different backgrounds.

A few studies have been conducted in this region regarding patient satisfaction in the primary care services in the UAE [1]; patient satisfaction in government health facilities in the State of Qatar [2] and quality of primary health care in Saudi Arabia [3]. There is very little research on how patients select their doctors and whether concordance between the nationality or ethnic background of the doctor and patient is important. This study hopes to fill the gap in this area.

\section{CULTURE AND HEALTHCARE}

There is plethora of literature on the importance of culture to health care. Most of the studies have been undertaken in the context of the U.S. health care system where both policy makers and health care providers are cognizant of the changing demographics of their society. The conclusion of studies [4], [5], [6], [7] and [8] is that culture and language play a significant role in determining the level of satisfaction and health outcomes of minorities in the United States.

\subsection{Dimensions of Culture}

Culture is not easy to define. It has many dimensions; and sensitivity to cultural differences has the inherent risk of stereotyping people. In the context of Dubai, the situation is more complex, since within a nationality or ethnic background there are many sub-groups with different languages and cultural backgrounds. "In sum, because health care is a cultural construct, arising from beliefs about the nature of disease and human body, cultural issues are actually central in the delivery of health services treatment and preventive interventions” [9].

\subsection{Health Care in the UAE}

Dubai is a multicultural city with a growing population. A unique phenomenon in this city is the fact that expatriate workers outnumber the nationals. Thus, both the health care providers and users largely comprise of non-locals. To date, the healthcare services are mainly staffed by foreign nationals: only 3.3 per cent of nursing staff are UAE nationals and approximately 10 per cent of them are doctors ${ }^{1}$.

\section{DATA SOURCE}

A questionnaire was developed and distributed to a random sample of the population. The number of questionnaires distributed was 130 and the number returned was 101 , giving a response rate of $78 \%$. Out of the completed questionnaires, 8 were incomplete and 93 responses were used for the analysis. Respondents aged 18 and above were eligible for this survey.

\subsection{Analysis of Survey Results}

The survey results provide some insight into how respondents select their doctors and how they perceive their own health.

\section{How do the respondents select their doctors?}

In the survey carried out, most of the respondents selected their doctors on the recommendation of family and friends. In the literature there is a considerable amount of emphasis on the significance of patients making informed choices

"In theory patients can play an important role in achieving optimal health by taking an active and informed role in treatment decisions and switching physicians if care is unsatisfactory” [10].

\footnotetext{
${ }^{1}$ United Arab Emirates Yearbook 2006

* PhD candidate SZABIST Faculty of Social Sciences asimashirazi@uowdubai.ac.ae
} 


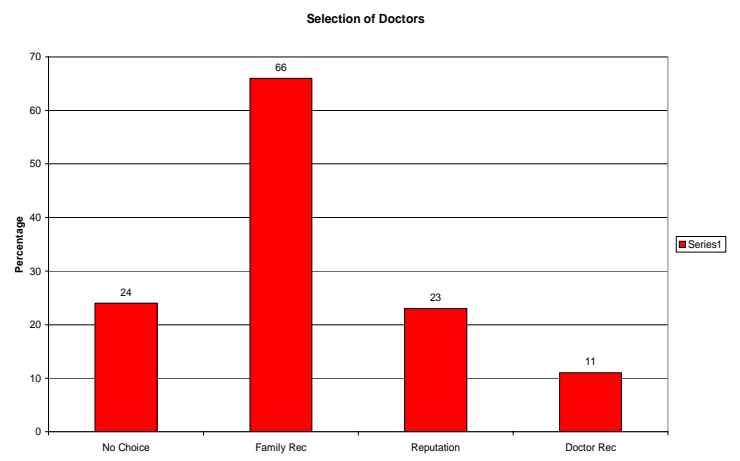

Figure 1: Source: Based on survey result by the author

The method of selection adopted by the respondents in Dubai confirms the results in other studies [10]. Twenty-two respondents (24 per cent) did not choose their own doctor. Of the remaining 73 respondents who selected their own doctors, 47 or 66 per cent selected their doctor on the recommendation of family and friends, 23 per cent on the basis of the reputation of the hospital or clinic where the doctor practiced and 11 per cent on the recommendation of another doctor.

Do patients select doctors on the basis of similar nationalities?

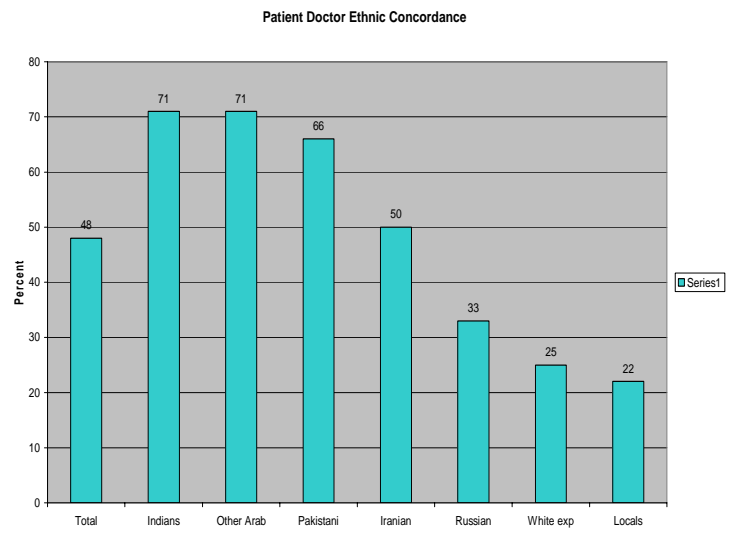

Figure 2: Source: Author based on survey result

Overall, 48 percent of the respondents went to doctors with the same nationality as themselves. In terms of percentage both Indians and other Arabs were the same (71\%). Only 22 percent of the locals went to local doctors. This reflects the under representation of local doctors in Dubai.
How do respondents perceive their health?

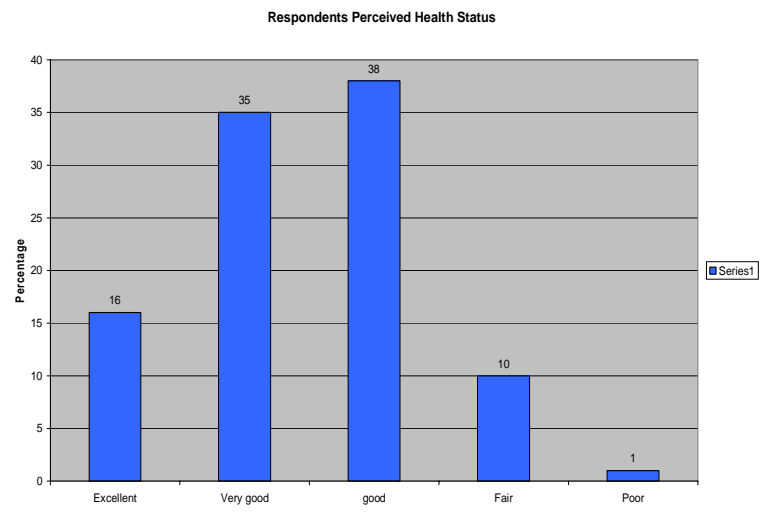

Figure 3: Source: Based on survey result by the author

Majority of the respondents perceived themselves as being in good to excellent health. A small minority considered themselves to be in fair to poor health. .

\subsection{An Econometric Model Explaining Patient Satisfaction}

The dependent variable was the satisfaction of respondents with their doctors. The dependent variable was defined as a dummy-binary choice. If the respondents were satisfied with the doctor, the response was Yes $=1$ and if they were not, the response was No $=0$. The logit model has been selected for estimation purposes.

$$
\begin{aligned}
& \mathrm{Li}=\left(\mathrm{P}_{\mathrm{i}} / 1-\mathrm{P}_{\mathrm{i}}\right)=\beta_{1}+\beta_{2} \text { how_select }+\beta_{3} \text { gov_pvt }+ \\
& \beta_{4} \text { payment }+\beta_{5} \text { same_nat }+\beta_{6} \text { age }+\beta_{7} \text { qualific }+\beta_{8} \\
& \text { male_fem }+\mathrm{u}
\end{aligned}
$$

The variables are:

$\left(\mathrm{P}_{\mathrm{i}} / 1-\mathrm{P}_{\mathrm{i}}\right)=$ probability that the individual will be satisfied with the doctor

how_select $=$ the method of selecting the doctors. There were four possibilities $0=$ no choice, $1=$ recommended by family and friends, 2 = recommended by another doctor and $3=$ reputation of the hospital or clinic

gov_pvt $=$ government hospital or clinic $=1$ and private hospital or clinic $=0$

payment $=$ self payment $=0$, insurance $=1$ and employer $=$ 2

same_nat $=$ if the nationality of the doctor is the same as the nationality of the respondent $=1$ if different $=0$

age $=$ different age groups were numbered from 1 to 6

Qualific $=$ qualification of respondent where not completed high school $=1$, completed high school $=2$, undergraduate degree $=3$ and post graduate degree $=4$ Male_Fem $=$ Male $=1$ and female $=0$

The equation was revised on the basis of the preliminary results and the non-significant variables were dropped.

The revised equation is given below: 
$\mathrm{Li}=\left(\mathrm{P}_{\mathrm{i}} / 1-\mathrm{P}_{\mathrm{i}}\right)=\beta_{1}+\beta_{2}$ same_nat $+\beta_{3}$ age $-\beta_{4}$ qualific

$+\mathrm{u}$ below:

The results of the revised equation are given in table 1

Convergence achieved after 6 iterations

Model 1: Logit estimates using the 93 observations 1-93

Dependent variable: Satisfied

Table 1

\begin{tabular}{|l|l|l|l|}
\hline VARIABLE & COEFFICIENT & STDERROR & T STAT \\
\hline const & 3.96260 & 1.62287 & 2.442 \\
\hline same_nat_ & 0.873619 & 0.686960 & $1.272^{+}$ \\
\hline Age & 0.914754 & 0.541761 & $1.688^{*}$ \\
\hline qualific & -1.23490 & 0.532242 & $-2.320^{* *}$ \\
\hline
\end{tabular}

+ Significant at $10 \%$ level

* Significant at $5 \%$ level

** Significant at $1 \%$ level

The coefficients of the logit model are estimated by the method of maximum likelihood estimation. For the logit model, convergence is usually achieved in 4 or 5 iterations. Furthermore, the form of the log-likelihood function for the logit model guarantees that different starting values will lead to unique maximum likelihood estimates. This attractive property makes the logit model an estimation method that can be used with some success. If the number of iterations exceeds 10 or 15 it could be an indication of multi-collinearity in the data set. However, the number of iterations in this case is 6 .

\subsection{Discussion of Results}

The coefficients of the logit model are estimated by the method of maximum likelihood estimation. A positive estimate of parameter $\beta$ in a binary choice model, such as logit indicates higher than average probability of the respondent being satisfied with the doctor. The parameters $\beta_{2}$ and $\beta_{3}$ for the dependent variables for same nationality and age are positive, indicating that the likelihood of the individual being satisfied with his or her doctor is greater if the doctor has the same nationality and background as the patient and the older the patient. Although statistically, the coefficient for same nationality is significant at $10 \%$ level; but this could be due to the small sample size. Studies undertaken in United States have concluded that ethnic concordance between patients and doctors can enhance the satisfaction level. [11] concludes that, among the black Americans and Hispanics, the satisfaction level increased if the physician was of the same ethnic background. Among Japanese Americans, the level of trust in physicians is greater if they are from the same ethnic background [12]. Some participants in their study [8] "preferred a provider of similar racial, ethnic and/or cultural background”.

The regression analysis shows the qualification of the respondent has the most significant impact on the level of satisfaction. The estimate of the parameter corresponding to qualification is negative. This implies that the probability of a person being satisfied with his or her doctor decreases with educational qualifications. The explanation for this outcome could be the fact that with education the respondents are more discerning and have higher expectations from the doctors.

\section{CONCLUSION AND RECOMMENDATIONS}

The survey results revealed some interesting features about the demand and supply side of health care in Dubai. There is a congruence of doctors and patients in terms of nationality. This result is possible because the providers and users of health services are non-local. Interestingly enough, the local population is in the same position as minorities would be in countries such as the United States. Overall, the level of satisfaction with doctors was high.

There is a further need to assess the cultural and linguistic needs of the growing population and provide training to health care providers both at the individual and institutional level to enable them to work effectively in cross-cultural situations.

\section{AREAS FOR FURTHER RESEARCH}

Due to limitations of time and resources, the number of participants in the survey was limited. An extension of this research to other emirates and a comparative study of Dubai and Abu Dhabi will be useful.

Another area of research would be to investigate the supply side and identify the current state of health care providers with respect to cultural competence.

\section{ACKNOWLEDGEMENT}

I would like to thank Dr. Amanat Ali Jalbani for his guidance and patience throughout the research and writing of this paper.

\section{REFERENCES}

[1] Margolis, S.A., et al. "Patient Satisfaction with Primary Care Services in the United Arab Emirates". International Journal for Quality in Health Care, 15, 3, May 2003.

[2] Al Kareem, A. A., et al. "Patient Satisfaction in Government Health Facilities in the State of Qatar". Journal of Community Health, page 349, New York, Volume 21, Issue 5, October 1996.

[3] Al-Ahmadi, H., Roland, M. "Quality of Primary Health Care in Saudi Arabia: A Comprehensive Review”, International Journal for Quality in Health Care, pages 331-346, Volume 17, Number 4, 2005. 
[4] Brach C., Fraser I. “Can Cultural Competency Reduce Racial and Ethnic Health Disparities? A Review and Conceptual Model”, Medical Care Research and Review 57, pages 181-217, Suppl 1, 2000.

[5] Andresen J. "Cultural Competence and Health Care: Japanese, Korean, and Indian Patients in the United States”, Journal of Cultural Diversity, 8, 4, pages 109-21, Winter 2001.

[6] Anderson L.M., Scrimshaw S.C., Fullilove M.T., et al. "Culturally Competent Healthcare Systems: A Systematic Review", American Journal of Preventive Medicine 24, pages 68-79, Suppl 3, April 2003.

[7] Hayes-Bautista D. E. "Research on Culturally Competent Healthcare Systems: Less Sensitivity, More Statistics", American Journal of Preventive Medicine, April 2003.

[8] Cook C.T., Koisoko-Lasaki O., O’Brien R. "Satisfaction With and Perceived Cultural Competency of Healthcare Providers: The Minority Experience", Journal of the National Medical Association 97, 8, pages 1078-87, Aug 2005.

[9] Katz Michael. "National Standards for Culturally and Linguistically Appropriate Services in Health Care”, U.S. Department of Health and Human Services, OPHS Office of Minority Health, Final Report, Washington D.C., March 2001.

[10] Harris K.M. "How Do Patients Choose Physicians? Evidence from a National Survey of Enrollees in Employment Related Health Plans", Health Services Research, pages 711-732, 38 (2), April 2003.

[11] Saha, S. et al. "Patient-Physician Racial Concordance and the Perceived Quality and Use of Health Care", Archives of Internal Medicine 1999, pages 159, 9971004.

[12] Tarn, D. M., et al. “Trust in One’s Physician: The Role of Ethnic Match, Autonomy, Acculturation, and Religiosity among Japanese and Japanese Americans”, Annals of Family Medicine 2005, pages 339-47, 3 (4). 